\title{
Incomplete Staging (cM)
}

National Cancer Institute

\section{Source}

National Cancer Institute. Incomplete Staging (CM). NCI Thesaurus. Code C159723.

A lack of clinical and/or pathology information to assign a cM stage conclusively. 